\title{
Revista Colombiana de

\section{Desenlaces clínicos en una cohorte de pacientes con síndrome coronario agudo y administración intracoronaria de tirofiban}

\author{
Julián Ochoa ${ }^{\mathrm{a}}$, Jorge G. Velásquez ${ }^{\mathrm{a}}$, Jorge A. Cedano ${ }^{\mathrm{c}}$, Camila Ospina $^{\mathrm{b}}$, \\ Lina Merchancano ${ }^{\mathrm{b}}$ y Diana C. Carrillo ${ }^{\mathrm{c}, *}$
}

\author{
a Hemodinamia, Servicio de Intervencionismo Vascular, Fundación Valle del Lili, Cali, Colombia \\ b Universidad ICESI, Cali, Colombia \\ c Centro de Investigaciones Clínicas, Fundación Valle del Lili, Cali, Colombia
}

Recibido el 12 de junio de 2014; aceptado el 10 de octubre de 2014

Disponible en Internet el 16 de marzo de 2015

\author{
PALABRAS CLAVE \\ Fibrinolíticos; \\ Inhibidores de \\ agregación \\ plaquetaria; \\ Síndrome coronario \\ agudo; \\ Intervencionismo \\ coronario percutáneo
}

\begin{abstract}
Resumen
Introducción: Los inhibidores de glicoproteína Ilb/Illa reducen los eventos cardiovasculares mayores adversos (MACE) en pacientes con síndrome coronario agudo.

Objetivos: Determinar la incidencia de MACE y desenlaces de seguridad en pacientes con síndrome coronario agudo llevados a intervención percutánea coronaria primaria y administración intracoronaria e intravenosa de tirofiban comparado con una población donde no se administró dicho medicamento.

Materiales y métodos: Cohorte prospectiva. Se incluyeron adultos con síndrome coronario agudo desde enero de 2010 hasta diciembre de 2012. Se realizó un modelo de riesgos proporcionales a un mes de seguimiento donde se evaluó el riesgo de MACE siendo la variable de interés el uso de tirofiban intracoronario e intravenoso.

Resultados: Se incluyeron 382 pacientes. Un 46\% (174 pacientes) recibieron tirofiban intracoronario. El promedio de edad fue 65 años vs. 60 años en los que no recibieron tirofiban $(p=0,00)$. La tasa de incidencia de MACE a un mes de seguimiento fue $13 / 1000$ eventos y 15/1000 eventos entre los que recibieron y no tirofiban respectivamente (Logrank test $=0,935$ ). El uso de tirofiban no se relacionó con menor incidencia de MACE (HR 1,09 95\% IC 0,72-1,65) ni con sangrado mayor a un mes de seguimiento $(1,72 \%$ vs. $2,88 \% \mathrm{p}=0,456)$.
\end{abstract}

\footnotetext{
* Autor para correspondencia.

Correo electrónico: diana_cristinac@hotmail.com (D.C. Carrillo).
} 


\section{KEYWORDS}

Fibrinolytic agents; Platelet aggregation inhibitors; Acute coronary syndrome; Percutaneous coronary intervention
Conclusiones: El uso de tirofiban intracoronario e intravenoso en pacientes con evento coronario agudo no se relacionó con menor incidencia de MACE ni eventos de sangrado en esta población, sin embargo, se deben tener en cuenta otras variables clínicas y de severidad del evento coronario no medidas que pudieron influir en los resultados.

(c) 2014 Sociedad Colombiana de Cardiología y Cirugía Cardiovascular. Publicado por Elsevier España, S.L.U. Este es un artículo Open Access bajo la licencia CC BY-NC-ND (http://creativecommons.org/licenses/by-nc-nd/4.0/).

\section{Clinical outcomes in cohor of patients with acute coronary syndrome and intracoronary tirofiban administration}

\begin{abstract}
Introduction: Glycoprotein IIb/IIla receptor inhibitors reduce major adverse cardiovascular events (MACE) in patients with acute coronary syndrome.

Objective: To determine the major adverse cardiovascular events and safety of intracoronary and intravenous tirofiban in patients with acute coronary syndrome with percutaneous coronary intervention $(\mathrm{PCl})$ compared to a group of patients without this medication.

Material and methods: Prospective cohort. Adults with acute coronary syndrome from January 2010 to December 2012 were included. A proportional hazard regression model after 1 month of follow up where the risk of MACE and the interest variable was intracoronary and intravenous tirofiban were assessed.

Results: 382 patients were included. 46\% (174 patients) received intracoronary tirofiban. The average age was 65 years vs. 60 years in the group of non-tirofiban users $(p=0.00)$. The rate of MACE at the first month was od 13/1000 events and 15/1000 events respectively in the group tirofiban and non-tirofiban (log rank test 0,935 ). The tirofiban use was neither related to a lower incidence of MACE (HR 1.09 95\% IC 0.721 .65 ) nor to major bleeding after the first month of follow up $(1.72 \%$ vs. $2.88 \%$ respectively, $p=0.456)$.

Conclusions: Intracoronary and intravenous tirofiban in patients with acute coronary syndrome was neither related to lower incidence of MACE nor to bleeding events; however, it should be taken into account that other clinical variables and the severity of unquantified coronary events might influence the results.

(c) 2014 Sociedad Colombiana de Cardiología y Cirugía Cardiovascular. Published by Elsevier España, S.L.U. This is an open access article under the CC BY-NC-ND license (http://creativecommons.org/licenses/by-nc-nd/4.0/).
\end{abstract}

\section{Introducción}

Los inhibidores de glicoproteína IIb/IIla (GPIIb/IIla) reducen los eventos cardiovasculares adversos mayores (MACE) en pacientes con síndromes coronarios agudos llevados a intervención coronaria percutánea primaria $(\mathrm{PCl})^{1-3}$. El papel de estos medicamentos en la fisiopatología de la enfermedad está relacionado con su efecto sobre la agregación plaquetaria debido a que la glicoproteína Ilb/ Illa actúa como puente sobre el fibrinógeno, lo que fortalece el trombo ${ }^{4-6}$.

Los inhibidores del receptor GP IIb/IIla (abiciximab, eptifibatide y tirofiban) son recomendados en las diferentes guías mundiales que orientan acerca del manejo de los pacientes con síndromes coronarios agudos y que van a ser llevados a intervenciones percutáneas coronarias ${ }^{7-9}$. Sin embargo, solamente se describe la administración intravenosa como la vía de preferencia a pesar que existe evidencia que demuestra que la administración en bolo intracoronario es segura y eficaz en la reducción de la carga trombótica, el tamaño de la zona infartada y los desenlaces clínicos adversos mayores para el caso de tirofiban $^{7-9}$. Esta estrategia de manejo fue ideada bajo la hipótesis de que una concentración sérica más alta dentro de las arterias coro- narias afectadas alcanzaría de forma expedita una mayor inhibición de la agregación plaquetaria y la formación de trombos ${ }^{10}$. Por lo anterior, se determinó la incidencia de desenlaces clínicos (MACE) y de seguridad (sangrados mayores) en una cohorte de pacientes con evento coronario agudo sometida a angioplastia coronaria primaria con implantación de stents y administración intracoronaria de tirofiban comparado con una población donde no se administró dicho medicamento.

\section{Materiales y métodos}

Se realizó un estudio observacional, analítico de una cohorte prospectiva en una unidad de intervencionismo cardiovascular de una institución de cuarto nivel de atención en Colombia donde se cuenta con un registro prospectivo institucional de los pacientes sometidos a angioplastia con intención de implante de stent coronario desde el año 1994. Se incluyeron los pacientes mayores de 18 años, sometidos a intervención percutánea coronaria por evento coronario agudo tipo infarto agudo del miocardio con elevación del segmento ST e infarto sin elevación del segmento ST en 
el período de enero de 2010 hasta diciembre de 2012. El protocolo institucional durante el intervencionismo cardiovascular en pacientes con evento coronario agudo incluye pretratamiento con heparina no fraccionada a una dosis de 5000 unidades de bolo intravenoso seguido de goteo intravenoso de 1000 unidades/hora por 24 horas, hasta alcanzar un control de tiempo de tromboplastina parcial (aPTT) de 1,5 a 2 veces el control del día o enoxaparina a una dosis de $1 \mathrm{mg} / \mathrm{kg}$ con dosis ajustada según la función renal. Igualmente incluye carga de ácido acetilsalicílico a una dosis de $300 \mathrm{mg}$ con dosis de mantenimiento de $100 \mathrm{mg}$ al día y carga de clopidogrel de $300 \mathrm{mg}$ a $600 \mathrm{mg}$ seguido de una dosis de $75 \mathrm{mg}$ al día. Después del cateterismo diagnóstico, la principal variable para la decisión de administrar tirofiban intracoronario fue la presencia de trombo intracoronario, definido según la estadificación del grupo TIMI para trombo intracoronario utilizando los grados uno a cinco por dos observadores diferentes. Posteriormente se realiza trombectomía aspiración manual con catéter dedicado coronario (Pegase Stiff ${ }^{\circledR}$, Arthesys) y luego administración de bolo intracoronario de tirofiban a dosis alta de $25 \mathrm{mcg} / \mathrm{kg}$ a través del catéter guía entre 1 a 3 minutos seguido de una infusión intravenosa a dosis de $0,075 \mathrm{mcg} / \mathrm{k} /$ minuto durante 18 horas. Luego se trata la arteria con angioplastia e implante de stent convencional o medicado si es el caso. En el laboratorio de cateterismo institucional no se dispone de stents coronarios autoexpandibles o con malla.

Se excluyeron los pacientes con infarto agudo del miocardio que no fueron intervenidos con stent por alguna causa, dosis de tirofiban desconocida, evento antes del año 2010, sin seguimiento en el primer mes posterior al procedimiento. Se recolectó la información de los antecedentes personales, la indicación del procedimiento, las características del procedimiento, los eventos adversos cardiovasculares mayores y de sangrado al egreso hospitalario y un mes después del procedimiento. Estos eventos fueron establecidos en la historia clínica durante la hospitalización posterior al evento, el reingreso hospitalario o durante las visitas de seguimiento al servicio de cardiología. Se definió evento adverso cardiovascular mayor como el combinado de infarto agudo del miocardio sin elevación del segmento ST (dolor torácico sugestivo asociado con un electrocardiograma sin elevación del ST y elevación de enzimas cardíacas en nuestro caso la troponina I en las primeras 24 horas), infarto agudo del miocardio con elevación del ST (dolor torácico sugestivo asociado con elevación del $\mathrm{ST} \geq 0,1 \mathrm{mV}$ en dos o más derivaciones contiguas que se consideran que sean de origen isquémico asociado con elevación de la troponina I en las primeras 24 horas del inicio de los síntomas), la revascularización del vaso tratado (necesidad de repetir el procedimiento intervencionista percutáneo o quirúrgico de cualquier segmento del vaso tratado previamente) y la muerte por todas las causas (cardiovasculares y no cardiovasculares). Los eventos de sangrado fueron definidos como sangrados mayores (sangrados fatales, intracraneal o con caída de la hemoglobina $>5 \mathrm{~g} / \mathrm{dl}$ o que requiriera transfusión de glóbulos rojos. Esta información se estratificó según el uso de tirofiban intravenoso e intracoronario vs. el no uso, este último indicado por alta carga trombótica y evento coronario agudo.

Se describieron las características demográficas, las clínicas y los antecedentes, se presentaron como los promedios y desviación estándar o las medianas y los rangos intercuartílicos según la normalidad de su distribución. Las variables categóricas se presentaron como proporciones. Se utilizaron pruebas de comparación según el uso de tirofiban intravenoso e intracoronario, $t$ de Student o Wilcoxon Mann Withney de acuerdo con el cumplimiento de supuestos para las variables numéricas y para el caso de las variables categóricas se realizó comparación con $\mathrm{Chi}^{2}$ o test exacto de Fisher, una prueba a dos colas y un valor de $\mathrm{p}$ significativo menor a 0,05. Se realizó un modelo de los riesgos proporcionales para determinar el peligro relativo de presentar un evento adverso cardiovascular mayor y de seguridad durante el primer mes de seguimiento según el uso de tirofiban intracoronario. Este modelo se ajustó por las variables estadísticamente diferentes en el análisis exploratorio así como por el tipo de stent utilizado, se realizó diagnóstico de los supuestos de proporcionalidad de la regresión. La sobrevida libre de eventos adversos cardiovasculares mayores y de sangrado a un mes se graficaron según el tipo de stent implantado en una curva de sobrevida de Kaplan Meier. Se compararon las dos curvas mediante log Rank Test.

\section{Resultados}

Se incluyeron 382 pacientes con evento coronario agudo de los cuales un 51\% presentaron infarto con elevación del segmento ST y un $49 \%$ infarto sin elevación del segmento ST. De estos 54,4\% (208 pacientes) no recibieron tirofiban y $45,5 \%$ recibieron tirofiban intracoronario e intravenoso. No se obtuvo información del tiempo de inicio de los síntomas y la administración de tirofiban, sin embargo, el 95\% de los pacientes tuvieron un tiempo puerta-balón menor de $90 \mathrm{~min}$. Se observó mayor uso de tirofiban intracoronario entre los pacientes con trombosis intracoronaria durante el procedimiento $(60,34$ vs. $7,73 \% p=0,000)$. El promedio de edad fue de 60 años (DE 11) y 65 años (DE 10) entre los que recibieron y no recibieron tirofiban intracoronario $(p=0,00)$, fueron de sexo masculino 77 y $70 \%$ de los casos respectivamente. El antecedente de tabaquismo se observó en 18,97 y $16,83 \%$ de los casos. La mitad de los pacientes tenían hipertensión arterial en ambos grupos (tabla 1). No hubo diferencias en las características sociodemográficas y antecedentes médicos entre los pacientes con infarto agudo del miocardio con elevación del segmento ST y sin elevación del segmento ST. En la tabla 2 se describen las características de ambos grupos.

Se observó una mayor incidencia de implante de stent convencional entre los pacientes que recibieron tirofiban intracoronario e intravenoso comparado con los que no recibieron este medicamento ( 40,23 vs. $51,69 \%$ respectivamente $p=0,02$ ). En el primer mes después del evento, la incidencia de MACE en los pacientes que recibieron tirofiban fue de 16,01 vs. $17,48 \%$ en los que no recibieron tirofiban (logrank $p=0,935$ ), (figs. 1 y 2 ). Se observaron seis eventos de sangrado $(2,88 \%)$ los pacientes que no recibieron tirofiban comparado con tres eventos $(1,72 \%)$ en los que recibieron tirofiban $(p=0,456)$. A un mes posterior al evento, después de realizar ajuste por variables estadísticamente significativas, el uso de tirofiban intracoronario e intravenoso no estuvo relacionado con menor probabilidad de presentar MACE (HR 1,08 95\% IC 0,7 2-1,65 p=0,68). Se observó que la historia de la diabetes mellitus se relacionó con mayor 
Tabla 1 Características demográficas, clínicas y del procedimiento de los pacientes con evento coronario agudo según uso de tirofiban

\begin{tabular}{|c|c|c|c|}
\hline \multirow[t]{2}{*}{ Variable } & \multicolumn{2}{|c|}{ Uso de tirofiban } & \multirow[t]{2}{*}{$\mathrm{p}$} \\
\hline & No (208) & Sí (174) & \\
\hline Edad $^{*}$ & $65(10)$ & $60(11)$ & 0,00 \\
\hline Sexo masculino & $147(70,67)$ & $134(77,01)$ & 0,16 \\
\hline \multicolumn{4}{|l|}{ Antecedentes médicos } \\
\hline Diabetes & $61(29,33)$ & $33(18,97)$ & 0,02 \\
\hline Hipertensión arterial & $125(60,10)$ & $100(57,47)$ & 0,60 \\
\hline ACV previo & $3(1,44)$ & $4(2,30)$ & 0,53 \\
\hline Infarto agudo del miocardio & $33(15,87)$ & $14(8,05)$ & 0,02 \\
\hline Revascularización miocárdica & $8(3,85)$ & $3(1,72)$ & 0,21 \\
\hline Angiolastia con stent & $14(6,73)$ & $8(4,6)$ & 0,37 \\
\hline Enfermedad arterial periférica & $5(2,40)$ & $1(0,57)$ & 0,15 \\
\hline Falla renal & $12(5,77)$ & $5(2,87)$ & 0,17 \\
\hline \multicolumn{4}{|l|}{ Procedimiento } \\
\hline \multicolumn{4}{|l|}{ No. vasos enfermos } \\
\hline 1 & $120(58,25)$ & $95(54,91)$ & 0,78 \\
\hline 2 & $52(53,06)$ & $46(26,59)$ & \\
\hline 3 & $34(16,50)$ & $32(18,50)$ & \\
\hline \multicolumn{4}{|l|}{ No. lesiones tratadas } \\
\hline 1 & $179(86,06)$ & $143(82,18)$ & 0,50 \\
\hline 2 & $26(12,5)$ & $29(16,67)$ & \\
\hline 3 & $3(1,44)$ & $2(1,15)$ & \\
\hline Estenosis inicial $^{* *}$ & $99(95-99)$ & $100(99-100)$ & 0,00 \\
\hline Lesión ostial & $13(6,28)$ & $25(14,37)$ & 0,00 \\
\hline Trombo presente & $16(7,73)$ & $105(60,34)$ & 0,00 \\
\hline \multicolumn{4}{|l|}{ Tipo stent } \\
\hline Convencional & $100(48,31)$ & $104(59,77)$ & 0,02 \\
\hline Medicado & $107(51,69)$ & $70(40,23)$ & \\
\hline
\end{tabular}

Medidas presentadas como número y proporción.

ACV: accidente cerebrovascular.

* : Promedio (desviación estándar).

** : Mediana (rango intercuartílico).

riesgo de presentar MACE (HR 1,63 95\% IC 1,11-2,40 p=0,01) y el uso de clopidogrel antes del procedimiento disminuyó el riesgo de presentar algún evento en el primer mes después del procedimiento (HR 0,52 95\% IC 0,34-0,78 $p=0,01$ ). Otros factores estadísticamente significativos en el exploratorio no se relacionaron con mayor o menor incidencia de MACE (tabla 3).

\section{Discusión}

Los estudios que se han realizado intentando estudiar el efecto de la administración intracoronaria de inhibidores de GP IIB/IIla en los síndromes coronarios agudos han demostrado beneficio en la destrucción de los trombos intracoronarios y mejoría en los parámetros hemodinámicos tales como el flujo TIMI coronario y blush del tejido miocárdico, especialmente en aquellos pacientes con una alta carga trombótica y reducción del flujo sanguíneo coronario como consecuencia de la alteración de la función microvascular ${ }^{11}$. Wu et al. en el 2008, demostró una reducción del MACE a 14 días sin observarse un efecto sostenido a 30 días con la administración intracoronaria de tirofiban ${ }^{12}$. Igualmente, Yang et al. en el 2007 encontraron un mejor flujo TIMI poscateterismo y una más rápida resolución de la elevación del segmento ST entre los pacientes con este tipo de infarto del miocardio $^{13}$. En el 2011, Friedland en un metaanálisis de 10 ensayos clínicos controlados (1590 pacientes) comparó tirofiban intracoronario vs. intravenoso en síndrome coronario agudo (SCA) sin encontrar diferencias en perfusión completa del vaso después de la $\mathrm{PCl}(\mathrm{RR} 1,0895 \%$ IC 1,01-1,15), sin embargo, el uso de tirofiban intracoronario se asoció con menor mortalidad a uno a tres meses (RR 0,45 95\% 0,23$0,90)$ pero no fue significativa a seis meses o más ${ }^{14}$. Kirma et al. en el 2012 presentó un estudio piloto donde se comparó el bolo intracoronario solo o con el bolo intravenoso y el mantenimiento en los 49 pacientes que fueron sometidos a $\mathrm{PCl}$ posterior a SCA. El desenlace principal fueron los índices hemodinámicos de perfusión microvascular medida al día 4-5 después del $\mathrm{PCl}$ y los secundarios fueron resolución del ST a 90 minutos, grado de blush miocárdico sin encontrarse diferencias significativas entre ambas vías de administración ${ }^{15}$. Otro estudio publicado por Zhu et al. en el 
Tabla 2 Características demográficas, clínicas y del procedimiento de los pacientes con infarto agudo del miocardio con elevación del segmento ST y sin elevación del segmento ST según uso de tirofiban

\begin{tabular}{|c|c|c|c|c|c|c|c|}
\hline \multicolumn{4}{|c|}{ Infarto agudo del miocardio con elevación del segmento ST } & \multicolumn{4}{|c|}{ Infarto agudo del miocardio sin elevación del segmento ST } \\
\hline \multirow[t]{2}{*}{ Variable } & \multicolumn{2}{|c|}{ Uso de tirofiban } & \multirow[t]{2}{*}{$\mathrm{p}$} & \multirow[t]{2}{*}{ Variable } & \multicolumn{2}{|c|}{ Uso de tirofiban } & \multirow[t]{2}{*}{$\mathrm{p}$} \\
\hline & No $(63)$ & Sí (132) & & & No $(143)$ & Sí (42) & \\
\hline Edad $^{*}$ & $63,4(10,8)$ & $59,4(11,12)$ & 0,02 & Edad $^{*}$ & $66(9,6)$ & $62(12,4)$ & 0,02 \\
\hline Sexo masculino & $52(82,54)$ & $104(78,79)$ & 0,54 & Sexo masculino & $93(65,03)$ & $30(71,43)$ & 0,44 \\
\hline Antecedentes médicos & & & & Antecedentes médicos & & & \\
\hline Diabetes & $19(30,16)$ & $29(19,7)$ & 0,11 & Diabetes & $41(28,67)$ & $7(16,67)$ & 0,12 \\
\hline Hipertensión arterial & $36(57,14)$ & $72(54,55)$ & 0,73 & Hipertensión arterial & $87(60,84)$ & $28(66,67)$ & 0,49 \\
\hline ACV previo & $1(1,59)$ & $1(0,76)$ & 0,59 & ACV previo & $2(1,4)$ & $3(7,14)$ & 0,04 \\
\hline Revascularización miocárdica & $1(1,59)$ & $1(0,76)$ & 0,59 & Revascularización miocárdica & $7(4,9)$ & $2(4,76)$ & 0,97 \\
\hline Angioplastia con stent & $4(6,35)$ & $7(5,30)$ & 0,76 & Angioplastia con Stent & $10(6,99)$ & $1(2,38)$ & 0,26 \\
\hline Enfermedad arterial periférica & $1(1,59)$ & $1(0,76)$ & 0,59 & Enfermedad arterial periférica & $3(2,10)$ & $0(0,00)$ & 0,34 \\
\hline Falla renal & $3(4,76)$ & $4(3,03)$ & 0,54 & Falla renal & $1(2,38)$ & $8(5,59)$ & 0,39 \\
\hline Procedimiento & & & & Procedimiento & & & \\
\hline No. vasos enfermos & & & & No. vasos enfermos & & & \\
\hline 1 & $33(53,23)$ & $70(53,44)$ & 0,53 & 1 & $86(60,56)$ & $25(59,52)$ & 0,97 \\
\hline 2 & $13(20,97)$ & $35(26,72)$ & & 2 & $38(26,76)$ & $11(26,29)$ & \\
\hline 3 & $16(25,81)$ & $26(19,85)$ & & 3 & $18(12,68)$ & $6(14,29)$ & \\
\hline No. lesiones tratadas & & & & No. lesiones tratadas & & & \\
\hline 1 & $56(88,89$ & $110(83,33)$ & 0,51 & 1 & $122(85,31)$ & $33(78,57)$ & 0,56 \\
\hline 2 & $7(11,11)$ & $21(15,91)$ & & 2 & $18(12,59)$ & $8(19,05)$ & \\
\hline 3 & 0 & $1(0,76)$ & & 3 & $3(2,10)$ & $1(2,38)$ & \\
\hline Estenosis inicial $^{* *}$ & 99 (95-99) & $100(99-100)$ & 0,00 & Estenosis inicial $^{* *}$ & $90(90-99)$ & $99(95-99)$ & 0,00 \\
\hline Lesión ostial & $8(12,9)$ & $23(17,42)$ & 0,42 & Lesión ostial & $5(3,5)$ & $2(4,76)$ & 0,71 \\
\hline Trombo presente & $13(20,97)$ & $85(64,39)$ & 0,00 & Trombo presente & $3(2,1)$ & $20(47,62)$ & 0,00 \\
\hline Tipo stent & & & & Tipo stent & & & \\
\hline Convencional & $39(61,9)$ & $78(59,09)$ & 0,71 & Convencional & $59(41,55)$ & $26(61,9)$ & 0,02 \\
\hline Medicado & $24(38,10)$ & $54(40,91)$ & & Medicado & $83(58,45)$ & $16(38,1)$ & \\
\hline
\end{tabular}

Medidas presentadas como número y proporción.

ACV: accidente cerebrovascular.

* : promedio (desviación estándar).

** : mediana (rango intercuartílico). 


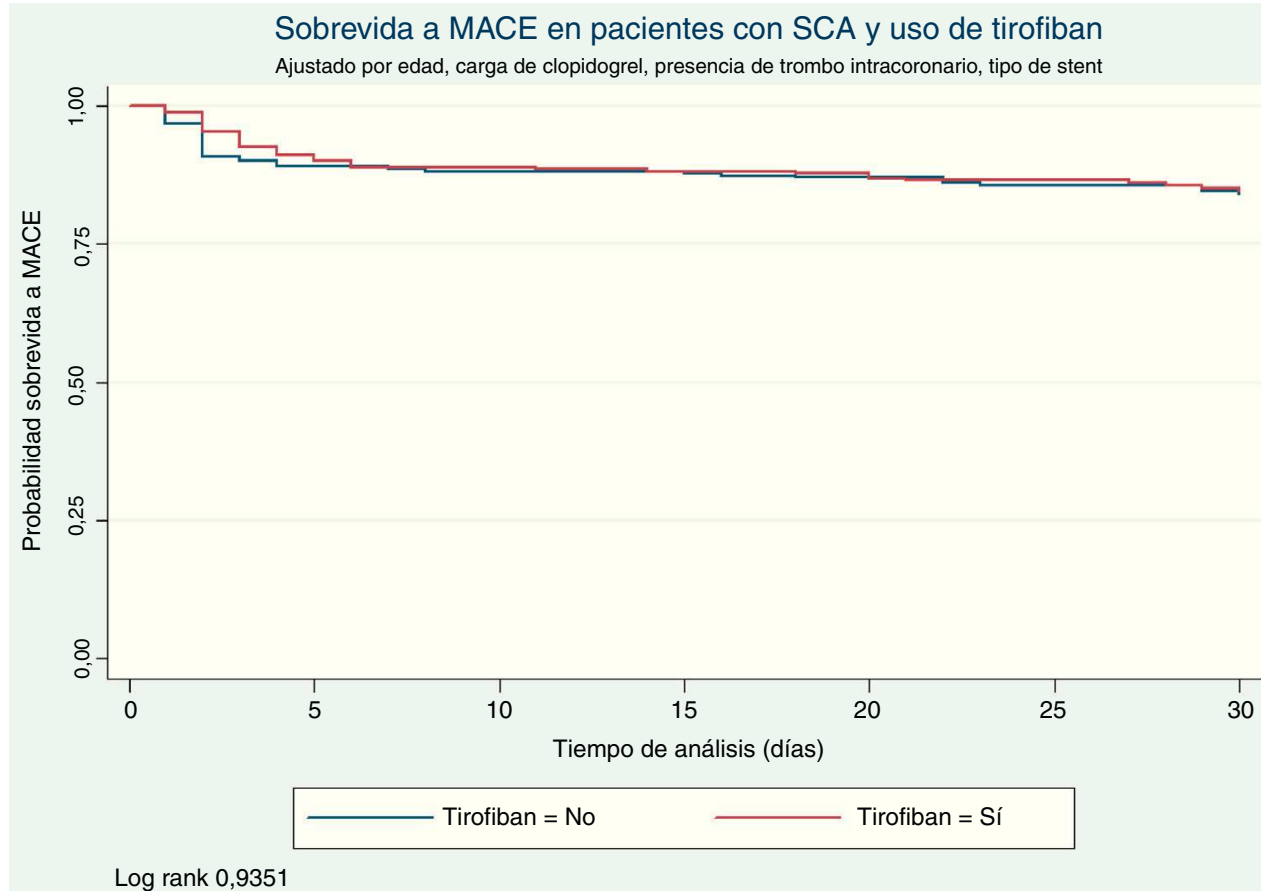

Figura 1 Sobrevida a eventos adversos cardiovasculares mayores en el primer mes de seguimiento según el uso de tirofiban.

2013 de 453 pacientes con STEMI sobre el uso de bolo intracoronario de tirofiban seguido de infusión por 24-36 horas con desenlace primario resolución del ST a 90 minutos después del procedimiento y secundarios MACE a 30 días y seis meses de seguimiento encontró mayor tasa de resolución del ST en 59 vs. 44,6\% $\mathrm{p}=0,002$, menor MACE a 30 días sin ser estadísticamente significativo $(3,1$ vs. $6,7 \% p=0,072)$, sin embargo, la sobrevida a 6 meses fue mayor en el grupo que recibió tirofiban intracoronario $(96,1$ vs. $90,6 \% p=0,020)$, aunque en este estudio se excluyeron los pacientes de shock cardiogénico con balón de contrapulsación aórtico, disfunción renal, inconsciente al ingreso y presencia de bloqueo de rama izquierda ${ }^{16}$. Para el 2013 se publicaron dos estudios por Zhu et al. y Chen et al. que incluyeron 530 y 203

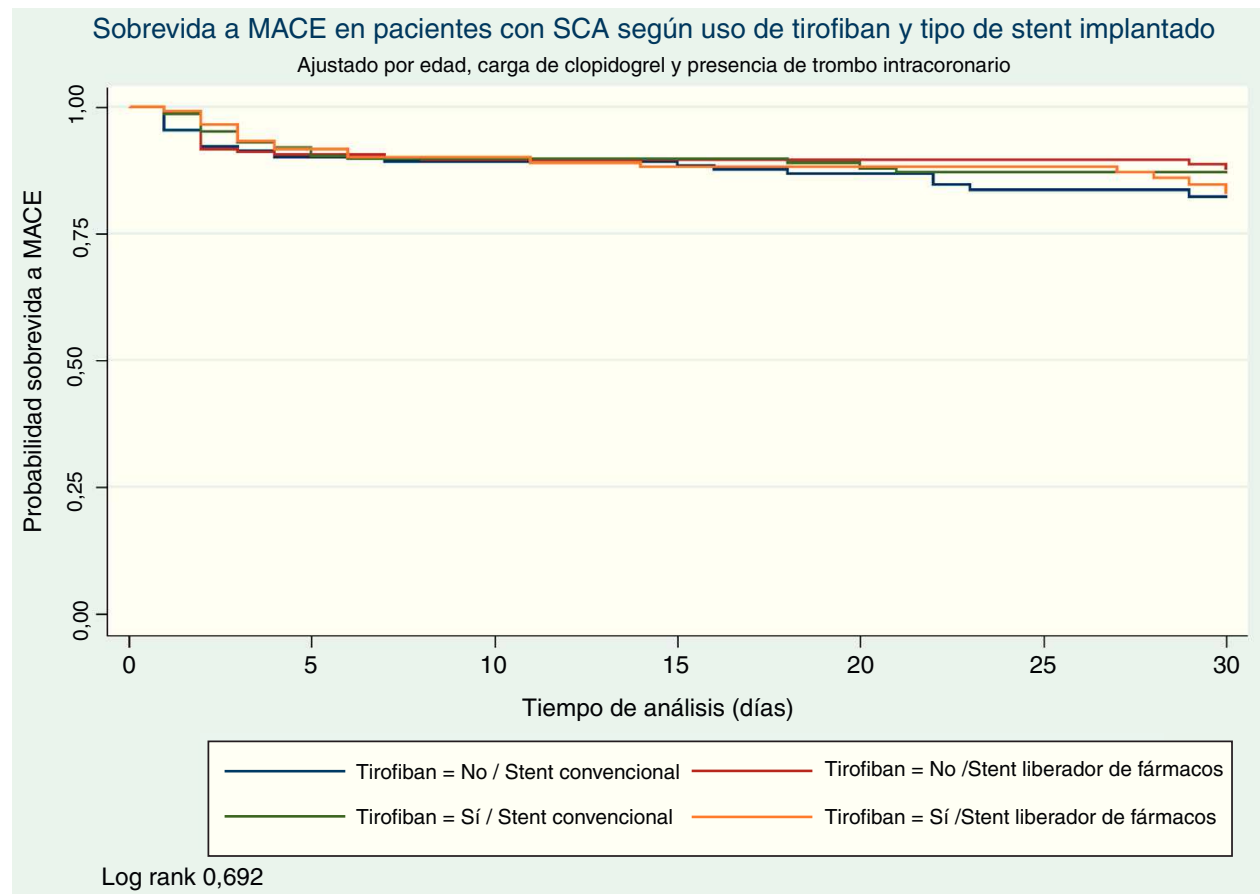

Figura 2 Sobrevida a 30 días de eventos adversos cardiovasculares mayores según el tipo de stent implantado y uso de tirofiban. SC: stent convencional; SLF: stent liberador de fármacos. 
Tabla 3 Modelo de riesgos proporcionales Cox de eventos adversos cardiovasculares mayores a un mes posterior al evento

\begin{tabular}{llll}
\hline Variable & Hazard Ratio & Intervalo de confianza a 95\% & $\mathrm{p}$ \\
\hline Uso de tirofiban & 1,08 & $0,72-1,64$ & 0,68 \\
Edad & 1,01 & $0,99-1,03$ & 0,06 \\
Diabetes mellitus & 1,63 & $1,11-2,39$ & 0,01 \\
Uso de clopidogrel & 0,52 & $0,34-0,78$ & 0,01 \\
Trombo intracoronario & 0,64 & $0,41-1,01$ & 0,06 \\
Uso de stent liberador de fármacos & 0,78 & $0,54-1,13$ & 0,19 \\
\hline
\end{tabular}

pacientes respectivamente con STEMI los cuales recibieron tirofiban intracoronario sin encontrar diferencias en MACE a 30 días y seis meses de seguimiento ${ }^{17,18}$.

En el presente trabajo no se encontraron diferencias significativas de la administración intracoronaria e intravenosa de tirofiban frente a los pacientes a los cuales no se administró dicho medicamento, sin embargo, este estudio tiene varias limitantes. La primera es el pequeño tamaño muestral obtenido con un bajo poder estadístico $(12,7 \%)$ lo cual podría influir en los resultados en el sentido que no se obtuvieron diferencias significativas cuando sí existen. Esto probablemente está relacionado con el desenlace buscado (incidencia de MACE) el cual requeriría un tamaño de muestra mucho mayor en comparación con la mejoría de las características angiográficas, sin embargo, consideramos que los desenlaces cardiovasculares tendrían un mayor impacto en la toma de decisiones clínicas. Así mismo, el diseño del estudio de tipo cohorte prospectiva, aunque se acerca un poco más al mundo real, podría a diferencia de los ensayos clínicos controlados tener variables no balanceadas en cada grupo que pudieran influir a favor o en contra de los resultados. Sin embargo, se realizó un análisis exploratorio de las variables sociodemográficas, las clínicas y la del procedimiento con el fin de detectar estas diferencias y controlarlas en el modelo de riesgos proporcionales, aun así pueden existir variables no medidas en el estudio que pudieran influir en el resultado. Otro factor fue el uso de carga de clopidogrel, ácido acetilsalicílico y anticoagulación que pudo disminuir el efecto del tirofiban en este subgrupo de pacientes. La otra situación a tener en cuenta es que en la mayoría de los estudios el desenlace principal son variables angiográficas y/o paraclínicas tales como flujo TIMI posprocedimiento, troponina, CKMB, disminución en milímetros del ST, variables que no se tuvieron en cuenta en el presente estudio. También se desconoce el tiempo entre inicio de síntomas y el inicio temprano o tardío de tirofiban aunque todos los pacientes recibieron este medicamento en sala de cateterismo una vez evidenciados hallazgos angiográficos. El tiempo puerta balón en nuestro estudio fue menor a 90 minutos en $95 \%$ de los casos. Hay que tener en cuenta en este último caso que todos los pacientes fueron intervenidos como $\mathrm{PCl}$ primaria. Asimismo, no se midieron ciertas características clínicas de severidad del evento isquémico que pudo estar relacionado con mayor o menor incidencia de eventos adversos cardiovasculares mayores a corto plazo, tales como presentar muerte súbita con evidencia de trombo intracoronario lo cual aumentó la probabilidad de requerir tirofiban intracoronario, shock cardiogénico, el no uso de carga de clopidogrel en este subgrupo de pacientes por estado neurológico los cuales han sido excluidos de los ensayos clínicos que evalúan el efecto de este medicamento.

\section{Conclusiones}

El uso de tirofiban intracoronario e intravenoso en pacientes con evento coronario agudo no se relacionó con menor incidencia de MACE ni eventos de sangrado en esta población, sin embargo, se deben tener en cuenta otras variables clínicas y de severidad del evento coronario no medidas que pudieron influir en los resultados. Se requieren más estudios que documenten la utilidad del uso local del tirofiban en el contexto de pacientes con síndromes coronarios agudos.

\section{Responsabilidades éticas}

Protección de personas y animales. Los autores declaran que para esta investigación no se han realizado experimentos en seres humanos ni en animales.

Confidencialidad de los datos. Los autores declaran que han seguido los protocolos de su centro de trabajo sobre la publicación de datos de pacientes.

Derecho a la privacidad y consentimiento informado. Los autores declaran que en este artículo no aparecen datos de pacientes.

\section{Conflicto de intereses}

Los autores declaran no tener ningún conflicto de intereses.

\section{Bibliografía}

1. Stone GW, Bertrand ME, Moses JW, Ohman EM, Lincoff AM, Ware $\mathrm{JH}$, et al. Routine upstream initiation vs deferred selective use of glycoprotein Ilb/llla inhibitors in acute coronary syndromes: the Acuity Timing trial. JAMA. 2007;297:591-602. Disponible en: http://jama.jamanetwork.com/article.aspx? articleid $=205521$

2. The Pursuit Trial Investigators. AT Inhibition of platelet glycoprotein IIb/IIla with eptifibatide in patients with acute coronary syndromes. N Engl J Med. 1998;339:436-43. Disponible en: http://www.nejm.org/doi/full/10.1056/NEJM199808133390704

3. Giugliano RP, White JA, Bode C, Armstrong PW, Montalescot G, Lewis BS, et al. Early versus delayed, provisional eptifibatide in acute coronary syndromes. $N$ Engl J Med. 2009;360:2176-90. Disponible en: http://www.nejm.org/ doi/full/10.1056/NEJMoa0901316 
4. The Prism-plus Study Investigators. Inhibition of the platelet glycoprotein Ilb/IIla receptor with tirofiban in unstable angina and non-Q-wave myocardial infarction. N Engl J Med. 1998;338:1488-97. Disponible en: http://www.nejm.org/ doi/full/10.1056/NEJM199805213382102

5. Bhatt DL, Topol EJ. Current role of platelet glycoprotein Ilb/IIla inhibitors in acute coronary syndromes. JAMA. 2000;284(12):1549-58. Disponible en: http://jama. jamanetwork.com/article. aspx?articleid $=193100$

6. Roffi M, Chew DP, Mukherjee D, Bhatt DL, White JA, Moliterno DJ, et al. Platelet glycoprotein IIb/IIla inhibition in acute coronary syndromes. Gradient of benefit related to the revascularization strategy. Eur Heart J. 2002;23:1441-8.

7. Steg G, James SK, Atar D, Badano LP, Lundqvist CB, Borger $M A$, et al. ESC Guidelines for the management of acute myocardial infarction in patients presenting with ST-segment elevation. Eur HHeart J. 2012;33:2569-619. Disponible en: http://www.escardio.org/guidelines-surveys/escguidelines/GuidelinesDocuments/Guidelines_AMI_STEMI.pdf

8. O'Gara PT, Kushner FG, Ascheim DD, Casey DE Jr, Chung MK, de Lemos JA, et al. 2013 ACCF/AHA Guideline for the management of ST-elevation myocardial infarction: a report of the American College of Cardiology Foundation/American Heart Association Task Force on Practice Guidelines. J Am Coll Cardiol. 2013;61(4):e78-140. Disponible en: http://content.onlinejacc. org/article. aspx?articleid $=1486115$

9. Anderson JL, Adams CD, Antman EM, Bridges CR, Califf RM, Casey DE, et al. 2012 ACCF/AHA Focused update incorporated into the ACCF/AHA 2007 guidelines for the management of patients with unstable angina/non-ST-elevation myocardial infarction: a report of the American College of Cardiology Foundation/American Heart Association Task Force on Practice Guidelines. J Am Coll Cardiol. 2013;61(23):e179-347. Disponible en: http://content.onlinejacc.org/article.aspx? articleid $=1217906$

10. De Vita M, Coluccia V, Burzotta F, Romagnoli E, Trani C. Intracoronary use of GP IIb/IIla inhibitors in percutaneous coronary interventions. Curr Vasc Pharmacol. 2012;10(4): 448-53.

11. Berger PB, Best PJ. Intracoronary glycoprotein IIb /IIla inhibitors from questioning the logic to weighing the data. J Am Coll Cardiol Intv. 2010;3:935-6. Disponible en: http: / / interventions. onlinejacc. org $/$ article. aspx?articleid=111194
12. Wu TG, Zhao Q, Huang WG, Wei JR, Chen SW, Zhao J, et al. Effect of intracoronary tirofiban in patients undergoing percutaneous coronary intervention for acute coronary syndrome. Circ J. 2008;72:1605-9. Disponible en: https://www.jstage. jst.go.jp/article/circj/72/10/72_CJ-08-0357/_pdf

13. Yang XC, Zhang DP, Wang LF, Xu L, Ge YG, Wang HS, et al. Effects of intracoronary or intravenous tirofiban administration in patients with acute ST-elevation myocardial infarction undergoing primary percutaneous coronary intervention. Zhonghua Xin Xue Guan Bing Za Zhi. 2007;35:517-22.

14. Friedland S, Eisenberg MJ, Shimony A. Meta-analysis of randomized controlled trials of intracoronary versus intravenous administration of glycoprotein Ilb/IIla inhibitors during percutaneous coronary intervention for acute coronary syndrome. Am J Cardiol. 2011;108:1244-51.

15. Kirma C, Erkol A, Pala S, Oduncu V, Dundar C, Izgi $A$, et al. Intracoronary bolus-only compared with intravenous bolus plus infusion of tirofiban application in patients with ST-elevation myocardial infarction undergoing primary percutaneous coronary intervention. Catheterization and cardiovascular interventions. 2012;79:59-67.

16. Zhu TQ, Qiu JP, Jin HG, Lu L, Shen J, Zhao LP, et al. Beneficial effects of intracoronary tirofiban bolus administration following upstream intravenous treatment in patients with ST-elevation myocardial infarction undergoing primary percutaneous coronary intervention: The ICT-AMI study. Int J Cardiol. 2013;165:437-43.

17. Zhu TQ, Zhang Q, Ding FH, Qiu JP, Jin HG, Jiang L, et al. Randomized comparison of intracoronary tirofiban vs urokinase as an adjunct to primary percutaneous coronary intervention in patients with acute ST-elevation myocardial infarction: results of the ICTUS-AMI trial. Chin Med J. 2013;126(16):3079-86. Disponible en: http://www.cmj.org/ $\mathrm{ch} /$ reader/view_abstract. aspx?volume=126\&issue=16\&start page $=3079$

18. Chen $Y$, Zhou $P$, Yan $H$, Zhao $H$, Song $L$, Chen $L$, et al. Impact of selective infarct-related artery infusion of tirofiban on myocardial reperfusion and bleeding complications in patients with acute myocardial infarction: The SUIT-AMI Trial. J Invasive Cardiol. 2013;25(8):376-82. Disponible en: http://www. invasivecardiology.com/articles/impact-selectiveinfarct-related-artery-infusion-tirofiban-myocardial-reperfusion -and-bleed 